\title{
CLINICAL CONDITIONS AND RISK FACTORS OF ACINETOBACTER BAUMANNII PRODUCING METALLO BETA-LACTAMASES AMONG HOSPITALIZED PATIENTS
}

\author{
Sulaiman A. Mohammed \\ Assistant Professor, Clinical Laboratory Technology, Thamar University, Yemen.
}

\section{ARTICLE INFO \\ Received: 21 September 2021 Revised: 06 November 2021 Accepted: 08 November 2021}

\section{Keywords:}

Metallo beta Lactamases, Acinetobacter baumannii, Risk Factors, Clinical Conditions.

Corresponding Author: Sulaiman A. Mohammed

Email:

alhusam212@yahoo.com Copyright (C) 2021 by author(s) and Journal of Scientific Research in Medical and Biological Sciences.

This work is licensed under the Creative Commons Attribution International License (CC BY 4.0). http://creativecommons.org/licenses/ by/4.0/

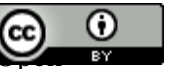

\section{o} ACcESS

\begin{abstract}
Purpose: The study aimed to determine the clinical conditions and risk factors associated with MBL produced by A.bumannii among hospitalized patients.

Subjects and Methods: The clinical samples were collected from inpatients and subcultured on routine culture media for growth. Identification of bacteria along with antimicrobial sensitivity testing was done by VITEK®2 compact (bioMerieux). Isolates that were resistant to Meropenem and/or Imipenem were followed to detection of MBL by using metallo- $\beta$-lactamases by imipenem EDTA combined disc test (IMP-EDTA CDT) method. Demographic and clinical data of each patient were collected in terms of the type of infection, hospital-stay, associated factors, and outcome till discharge.

Results: A number of 73(86.9\%) isolates of A.baumannii were resistant to carbapenem. out of 73 carbapenem-resistant isolates, $64(87.7 \%)$ were found to be $M B L$ positive. The patients with age more than 60 years i.e. $35.9 \%(23 / 64)$ were found to be more common in MBL positive isolates of A. baumannii. The difference in the distribution of $M B L$ positive and $M B L$ negative cases with endotracheal intubation and in Surgery during the last 30 days of incubation was found to be statistically significant. The mortality rate of patients infected by MBL positive isolates of A.baumannii was $12.5 \%$.

Conclusion: The MBL positive strains among carbapenem-resistant isolates of A.baumannii were high. endotracheal intubation and Surgery during the last 30 days were independently associated with MBL positive cases.
\end{abstract}

\section{INTRODUCTION}

The word Acinetobacter came from the Greek word 'akinetose' which means non-motile. The first isolate of Acinetobacter was reported in 1911 from the soil by Beijerinck (Almasaudi, 2018). Since 1986, Acinetobacter baumannii has established itself as the most frequent species of Acinetobacter genus in clinical samples and has become a formidable pathogen responsible for a number of nosocomial infection outbreaks (Cisneros \& Rodríguez-Baño, 2002). The reports of multidrug-resistant isolates A.bumannii have increased during the last decade, probably due to the increasing use of antimicrobial agents (Mohammed \& Singh, 2019). 
A. baumannii becomes a common organism in a hospital environment. The most important locations in medical care where A. baumannii has been found include urine collection bags, a container of distilled water, moist bedding articles, a multidose vial of medication, intravenous nutrition, and inadequately sterilized reusable arterial pressure transducers (Paterson, 2006).

Length of stay in the hospital particularly in the intensive care unit (ICU) and indwelling devices like mechanical ventilation, primary venous catheterization, and urinary catheterization are some of the common risk factors which are responsible for the development of carbapenem-resistant isolates of A.baumannii (Lee et al., 2017; Ellis et al.,2015). Identification of the risk factors associated with infection by strains producing carbapenemases is necessary for better management and prevention (Hong et al., 2015).

The present study aimed to identify the clinical conditions and risk factors of MBL among isolates of A.baumannii from various clinical specimens among hospitalized patients of Shree Krishna Hospital, Karamsad.

\section{METHODOLOGY}

A cross-sectional study was conducted from 30 September 2017 to 28 February 2018. The study was approved by the Human Research Ethics Committee (HREC) of H.M Patel Centre for Medical Care and Education, India. The clinical samples like pus, endotracheal aspirates, blood, sputum, body fluid, etc received in the Microbiology laboratory from hospitalized patients for culture \& sensitivity. Identification of A.baumannii along with antimicrobial sensitivity testing was done by $\mathrm{VITEK}^{\circledR} 2$ Compact microbiology systems (bioMerieux, France). Isolates that were resistant to Meropenem and/or Imipenem were followed to detection of metallo- $\beta$-lactamases (MBL) by using metallo- $\beta$-lactamases by Imipenem EDTA combined disc test (IMP-EDTA CDT) method. Demographic and clinical data of each patient were collected in terms of the type of infection, hospital stay, associated factors, and outcome till discharge. Statistical analysis was performed using the statistical package for social science (SPSS) software version 20.0. The results were presented as descriptive statistics in terms of relative frequency. A Chi-square test was used to analyze the results wherever needed. A Pvalue of $<0.05$ was considered statistically significant.

\section{RESULTS AND DISCUSSION}

Table 1: Distribution of MBL status in relation to carbapenem-resistant isolates of $A$. baumannii $(\mathrm{n}=73)$.

Types of Isolates

MBL Positive

MBL Negative

Total
A. baumannii $\mathrm{n}=73(\%)$

64(87.7)

$9(12.3)$

73(100)

Table 2: Distribution of Age group/years in relation to MBL status in total isolates of A. baumannii $(\mathrm{n}=84)$.

\begin{tabular}{ccc}
\hline Age group/years & MBL Positive $\mathbf{n = 6 4}(\boldsymbol{\%})$ & MBL Negative $\mathbf{n}=\mathbf{2 0}(\%)$ \\
\hline$\leq 1-20$ & $14(21.8)$ & $3(15.0)$ \\
\hline
\end{tabular}




\begin{tabular}{ccc}
\hline $21-40$ & $14(21.8)$ & $9(45.0)$ \\
$41-60$ & $13(20.3)$ & $2(10.0)$ \\
$>60$ & $23(35.9)$ & $6(30.0)$ \\
Total & $64(100)$ & $20(100)$ \\
\hline
\end{tabular}

During the study period, 2843 non-duplicated clinical specimens were processed for culture and sensitivity tests that yielded $947(33.3 \%)$ pathogenic organisms. A number of $737(77.8 \%)$ out of 947 were found to be gram-negative bacteria of which $235(31.9 \%)$ were no fermenting gram-negative bacteria (NFGNB). The prevalence of $A$. baumannii was $8.9 \%$ out of total pathogenic bacteria, $11.4 \%$ out of gram-negative bacteria, and $35.7 \%$ out of NFGNB.

As seen in table number 1, a number of 73(86.9\%) isolates of A.baumannii were resistant to carbapenem. Out of 73 carbapenem-resistant isolates, 64(87.7\%) were found to be MBL positive. the patients with age more than 60 years i.e. Also, 35.9\% (23/64) were found to be more common in MBL positive isolates of A. baumannii followed by age group of 1-20 and 21-40 years i.e. 14 (21.8\%, each), table 2 .

Table 3, Comparison of clinical conditions, co-morbidities, Indwelling/devices and outcome of patients with MBL positive and MBL negative isolates of A.baumannii.

\begin{tabular}{lllc}
\hline Parameters & $\begin{array}{l}\text { MBL Positive } \\
\mathrm{n}=64(\%)\end{array}$ & $\begin{array}{l}\text { MBL Negative } \\
\mathrm{n}=20(\%)\end{array}$ & P-value \\
\hline Age, mean+_SD (y) & $46.67+26.03$ & $41.60+21.39$ & 0.431 \\
Clinical Condition & $10(15.6)$ & $3(15.0)$ & $>0.999$ \\
Acute febrile/ septicemias & & & \\
Soft tissue infection & $10(15.6)$ & $2(10.0)$ & 0.722 \\
Respiratory tract infection & $38(59.4)$ & $11(55.0)$ & 0.798 \\
Trauma/head injury & $3(4.7)$ & $2(10.0)$ & 0.588 \\
Surgical conditions & $3(4.7)$ & $2(10.0)$ & 0.588 \\
Co- morbidities & & & \\
Diabetes mellitus & $10(15.6)$ & $0(0.0)$ & 0.108 \\
Neoplastic conditions & $15(23.4)$ & $7(35.0)$ & 0.383 \\
Hypertension & $4(6.2)$ & $2(10.0)$ & 0.625 \\
Using steroid & $6(9.4)$ & $1(5.0)$ & $>0.999$ \\
Surgery during last 30 days & $18(28.2)$ & $1(5.0)$ & 0.033 \\
Lung dysfunction & $16(25.0)$ & $4(20.0)$ & 0.770 \\
Cardiac dysfunction & $2(3.1)$ & $0(0.0)$ & 0.999 \\
Liver dysfunction & $8(12.5)$ & $4(20.0)$ & 0.467 \\
Kidney dysfunction & $11(17.2)$ & $2(10.0)$ & $>0.999$ \\
Indwelling/devices & & & 0.718 \\
Ventilator & $48(75.0)$ & $15(75.0)$ & 0.251 \\
Renal dialysis & $8(12.5)$ & $3(15.0)$ & $* 0.046$ \\
Tracheostomy & $19(29.7)$ & $3(15.0)$ & $>0.999$ \\
Endotracheal intubation & $22(34.3)$ & $2(10.0)$ & \\
Bronchoscopy & $5(7.8)$ & $1(5.0)$ & 0.238 \\
Duration stay/day & & & 0.146 \\
0-2 & $2(3.1)$ & $2(1.0)$ & 0.756 \\
3-5 & $7(10.9)$ & $5(25.0)$ & \\
6-10 & $13(20.3)$ & $5(25.0)$ & \\
11-30 & $26(20.6)$ & $5(25.0)$ & \\
\hline & & & \\
\hline
\end{tabular}

13 | 


\begin{tabular}{|c|c|c|c|}
\hline$>30$ & $16(25.0)$ & $3(15.0)$ & 0.541 \\
\hline \multicolumn{4}{|c|}{ Clinical outcome } \\
\hline Improved & $40(62.5)$ & $15(75.0)$ & 0.421 \\
\hline DAMA & $16(25.0)$ & $4(20.0)$ & 0.769 \\
\hline Expired & $8(12.5)$ & $1(5.0)$ & 0.679 \\
\hline
\end{tabular}

As can be seen from Table 3, the most common clinical conditions in MBL positive cases of A.baumannii were respiratory tract infection (59.4\%) followed by acute febrile/ septicemias, and soft tissue infection (15.6\%, each).

Surgery during the last 30 days was the commonest co-morbidity in MBL positive isolates of A.baumannii i.e. $28.2 \%(18 / 64)$ followed by lung dysfunction in $25.0 \%(16 / 64)$ and neoplastic conditions in $23.4 \%$ (15/64). MBL negative strains in the surgery during the last 30 days were found to be statistically significant with a p-value of 0.033 .

The highest 48(75.0\%) frequency of indwelling /devices in MBL positive isolates of $A$. baumannii were of ventilator followed by endotracheal intubation and tracheostomy i.e. $48(75.0 \%), 22(34.3 \%)$, and 19(29.7\%) respectively. The difference in the distribution of MBL positive and MBL negative strains in endotracheal intubation was found to be statistically significant with a p-value of 0.046 .

The highest number of MBL positive cases had a stay of 11-30 days followed by more than 30 days of hospital stay i.e $26(20.6 \%)$ and 16 (25.0\%) respectively. The distribution of DAMA and expired cases were found to be more percentage in MBL positive isolates than MBL negative isolates.

\section{Discussion}

The prevalence of Metallo beta-lactamase among carbapenem-resistant isolates of A. baumannii in this study was found to be $87.8 \%$. This is higher than the previous studies reported in India by Gupta V et al (2006), Karthika RU et al (2009) and Singla et al (2013) i.e. 7.5\%, 70.9\% and 57.5\%, respectively (Gupta, Datta, \& Chander, 2006; Singla et al., 2013). Also, the rate is higher when compared to $6.2 \%$ by Mesli et al (2013) from Algeria (Mesli et al., 2013), 3.2\% by Ryoo NH et al (2010) from Korea (Mesli et al., 2013), and 27.1\% by El-Ageery SM et al (2014) from Saudi Arabia (El-Ageery \& Al-Hazmi, 2014). But the rate is lower when compared to the percentage of $96.0 \%$ by Aksoy MD et al. (2015) from Turkey (Aksoy et al., 2015) and similar to $86.86 \%$ by Noori M et al (2014) from Iran ( Noori et al., 2014).

In this study, the significant difference in MBL positive and MBL negative cases of A.baumannii was identified only in endotracheal intubation $(34.3 \%$ Vs $10.0 \% \mathrm{p}=$ $0.046)$ and other devices such as ventilator use $(75.0 \%$ Vs $75.0 \% \mathrm{p}=>0.999)$, renal dialysis $(12.5 \% \mathrm{Vs} 15.0 \% \mathrm{p}=0.718)$, tracheostomy $(29.7 \% \mathrm{Vs} 15.0 \% \mathrm{p}=0.251)$ and bronchoscopy $(7.8 \%$ Vs $5.8 \% \mathrm{p}=>0.999)$. To the best of the author's knowledge, there is no previous study reporting the association between clinical devices in 
patients and the infection with MBL positive isolates of A. Baumannii. However, Zhou $\mathrm{H}$ et al (2019) in their study on multidrug resistance A. baumannii (MDRAB) found use of clinical devices to be statistically significant in comparison to nonmultidrug resistance $A$. baumannii (Non-MDRAB) i.e. central vein catheterization $(74.8 \%$ Vs $62.5 \% \quad \mathrm{p}=<0.001)$, urinary catheter $(91.2 \% \quad$ Vs $32.8 \% \quad \mathrm{p}=<0.001)$ mechanical ventilation $(78.5 \%$ Vs $18.8 \% \mathrm{p}=0.0257)$ and tracheal intubation $(79.9 \%$ Vs 21.9\% $\mathrm{p}=<0.001$ ) (Zhou et al., 2019).

In this study, only surgical procedures done before 30 days (28.2\% Vs $5.0 \% \mathrm{p}=0.033$ ) were found to be significance as compared to MBL negative, other co-morbidities as as: diabetes mellitus (15.6\% Vs $0.0 \% \mathrm{p}=0.108)$, neoplastic conditions $(23.4 \%$ Vs $35.0 \% \mathrm{p}=0.383$ ), hypertension $(6.2 \%$ Vs $10.0 \% \mathrm{p}=0.625)$, use of steroids $(9.4 \%$ Vs $5.0 \% \mathrm{p}=>0.999)$, cardiac dysfunction $(3.1 \% \mathrm{Vs} 0.0 \% \mathrm{p}=>0.999)$, kidney dysfunction $(17.2 \% \mathrm{Vs} 10.0 \% \mathrm{p}=0.724)$, liver dysfunction (12.5\% Vs $20.0 \% \mathrm{p}=0.467)$, lung dysfunction $(25.0 \%$ Vs $20.0 \% \mathrm{p}=0.770)$.

More importantly, there is a dearth of studies on co-morbidities in cases infected by MBL positive isolates of A. Baumannii. Huang ST et al (2012) reported that the co-morbidities observed in carbapenem-resistant and carbapenem susceptible isolates of A. baumannii were steroid use (43.5\% Vs 36\% p=0.372), hypertension (33.9\% Vs 32.3\% p=0.95), chronic kidney disease (25.8\% Vs 17.1\% p=0.197), diabetes mellitus (24.2 Vs 24.4 p=>0.999), Chronic obstructive pulmonary disease COPD (22.6\% Vs $15.9 \% \mathrm{p}=0.324)$ and liver cirrhosis $(1.6 \% \mathrm{Vs}$ $6.1 \mathrm{p}=0.297$ ) and were not significantly associated ( Huang et al., 2012).

In this study, the mortality rate of a patient infected by MBL positive isolates of $A$. baumannii was $12.5 \%$. Pandy Y et al (2016) reported that the mortality rate of patients infected by A. baumannii was $27.2 \%$ which is higher than our findings ( Pandya et al., 2016).

\section{CONCLUSION}

The MBL positive strains among carbapenem-resistant isolates of A.baumannii were high. endotracheal intubation and Surgery during the last 30 days were independently associated with MBL positive cases. The distribution of DAMA and expired cases were found to be more prevalent in MBL positive isolates than in MBL negative isolates. The results of this study could be useful for clinicians and the infection control system in hospitals.

\section{CONFLICTS OF INTEREST}

There are no conflicts of interest

\section{FUNDING}

No Funds were received for completing this study.

\section{REFERENCES}

Almasaudi, S. B. (2018). Acinetobacter spp. as nosocomial pathogens: Epidemiology and resistance 
features. Saudi journal of biological sciences, 25(3), 586-596. https://doi.org/10.1016/j.sjbs.2016.02.009

Cisneros, J. M., \& Rodríguez-Baño, J. (2002). Nosocomial bacteremia due to Acinetobacter baumannii: epidemiology, clinical features and treatment. Clinical Microbiology and Infection, 8(11), 687-693. https://doi.org/10.1046/j.1469-0691.2002.00487.x

Mohammed, S. A., \& Singh, S. P. (2019). Molecular Epidemiology of Metallo Beta Lactamases in Acinetobacter baumannii at a Tertiary Care Hospital. International Journal of Medical and Biomedical Studies, 3(8), 243-249. https://doi.org/10.32553/ijmbs.v3i8.500

Paterson, D. L. (2006). The epidemiological profile of infections with multidrug-resistant Pseudomonas aeruginosa and Acinetobacter species. Clinical infectious diseases, 43, S43-S48. https://doi.org/10.1086/504476

Lee, C. H., Su, T. Y., Ye, J. J., Hsu, P. C., Kuo, A. J., Chia, J. H., \& Lee, M. H. (2017). Risk factors and clinical significance of bacteremia caused by Pseudomonas aeruginosa resistant only to carbapenems. Journal Of Microbiology, Immunology and Infection, 50(5), 677-683. https://doi.org/10.1016/j.jmii.2015.06.003

Ellis, D., Cohen, B., Liu, J., \& Larson, E. (2015). Risk factors for hospital-acquired antimicrobialresistant infection caused by Acinetobacter baumannii. Antimicrobial resistance and infection control, 4(1), 1-5. https://doi.org/10.1186/s13756-015-0083-2

Hong DJ, Bae IK, Jang I-H, Jeong SH, Kang H-K, Lee K. 92015). Epidemiology and characteristics of metallo- $\beta$-lactamase-producing Pseudomonas aeruginosa. Journal of Infect Chemother, 47(2), 81-97. https://doi.org/10.3947/ic.2015.47.2.81

Gupta, V., Datta, P., \& Chander, J. (2006). Prevalence of metallo- $\beta$ lactamase (MBL) producing Pseudomonas spp. and Acinetobacter spp. in a tertiary care hospital in India. Journal of Infection, 52(5), 311-314. https://doi.org/10.1016/j.jinf.2005.08.013

Karthika, R. U., Rao, R. S., Sahoo, S., Shashikala, P., Kanungo, R., Jayachandran, S., \& Prashanth, K. (2009). Phenotypic and genotypic assays for detecting the prevalence of metallo- $\beta$-lactamases in clinical isolates of Acinetobacter baumannii from a South Indian tertiary care hospital. Journal of medical microbiology, 58(4), 430-435. https://doi.org/10.1099/jmm.0.002105-0

Singla, P., Sikka, R., Deep, A., \& Chaudhary, U. (2013). Phenotypic Detection And Prevalence Of Metallo B-Lactamases (Mbls) In Carbapenem Resistant Isolates of Acinetobacter Species At A Tertiary Care Hospital in North India. Int $J$ Pharm Med Bio Sci, 2, 85-91. https://doi.org/20150412031531190

Mesli, E., Berrazeg, M., Drissi, M., Bekkhoucha, S. N., \& Rolain, J. M. (2013). Prevalence of carbapenemase-encoding genes including New Delhi metallo- $\beta$-lactamase in Acinetobacter species, Algeria. International Journal of Infectious Diseases, 17(9), e739-e743. https://doi.org/10.1016/j.ijid.2013.02.024

Ryoo, N. H., Ha, J. S., Jeon, D. S., \& Kim, J. R. (2010). Prevalence of Metallo- $\beta$-lactamases in Imipenem-non-susceptible Pseudomonas aeruginosa and Acinetobacter baumannii. Korean Journal of Clinical Microbiology, 13(4), 169-172. https://doi.org/10.5145/KJCM.2010.13.4.169

El-Ageery, S. M., \& Al-Hazmi, S. S. (2014). Microbiological and molecular detection of VIM-1 metallo beta lactamase-producing Acinetobacter baumannii. European Review for Medical and Pharmacological Sciences, 18(7), 965-970. https://doi.org/10.5145/KJCM.2010.13.4.169

Aksoy, M. D., Çavuşlu, Ş., \& Tuğrul, H. M. (2015). Investigation of metallo beta 
lactamases and oxacilinases in carbapenem resistant Acinetobacter baumannii strains isolated $\begin{array}{lllll}\text { from inpatients. Balkan medical journal, } & 32(1),\end{array}$ https://doi.org/10.5152/balkanmedj.2015.15302

Noori, M., Karimi, A., Fallah, F., Hashemi, A., Alimehr, S., Goudarzi, H., \& Aghamohammad, S. (2014). High prevalence of metallo-beta-lactamase producing Acinetobacter baumannii isolated from two hospitals of Tehran, Iran. Archives of Pediatric Infectious Diseases, 2(3), 15. https://doi.org/10.5812/pedinfect.15439

Zhou, H., Yao, Y., Zhu, B., Ren, D., Yang, Q., Fu, Y., ... \& Zhou, J. (2019). Risk factors for acquisition and mortality of multidrug-resistant Acinetobacter baumannii bacteremia: A retrospective study from a Chinese hospital. Medicine, 98(13). https://doi.org/10.1097/MD.0000000000014937

Huang, S. T., Chiang, M. C., Kuo, S. C., Lee, Y. T., Chiang, T. H., Yang, S. P., ... \& Fung, C. P. (2012). Risk factors and clinical outcomes of patients with carbapenem-resistant Acinetobacter baumannii bacteremia. Journal of Microbiology, Immunology and Infection, 45(5), 356-362. https://doi.org/10.1016/j.jmii.2011.12.009

Pandya, Y., Singh, S., Badodariya, D., \& Shethwala, N. (2016). Metallo-B-Lactamase Producing Clinical Isolates of Acinetobacter baumannii and Pseudomonas aeruginosa in A Teaching Hospital of Rural Gujarat-India. National Journal of Integrated Research in Medicine, 7(6). https://doi.org/10.4103/0255-0857.42035 